RICYDE. Revista Internacional de Ciencias del Deporte doi: $10.5232 /$ ricyde

Rev. int. cienc. deporte

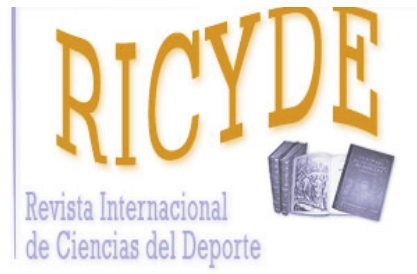

RICYDE. Revista Internacional de Ciencias del Deporte VOLUMEN XIV - AÑO XIV

Páginas:205-218 ISSN:1885-3137

Número: 53 - Julio - 2018

\title{
Impacto de dos modalidades de entrenamiento con electroestimulación global de cuerpo completo sobre la composición corporal en corredores recreacionales durante periodos de desentrenamiento deportivo
}

\section{Impact of two whole-body electromyostimulation training modalities on body composition in recreational runners during endurance training cessation}

\author{
Francisco José Amaro-Gahete, Alejandro De la O, Lidia Robles-González, \\ Manuel Joaquín Castillo y Ángel Gutiérrez \\ Universidad de Granada. España
}

\section{Resumen}

La electroestimulación de cuerpo completo (WB-EMS) es una metodología de entrenamiento novedosa cuyo efecto sobre la composición corporal no ha sido estudiado en profundidad en población joven y físicamente activa. El objetivo del estudio fue determinar el efecto de dos programas de entrenamiento con WB-EMS sobre la composición corporal, medida a través del Índice de Masa Corporal (IMC), masa grasa (MG), masa libre de grasa (MLG) y densidad mineral ósea (DMO) en corredores recreacionales entrenados durante un periodo de cese parcial en su entrenamiento convencional y comparar el efecto de dos programas de entrenamiento con WB-EMS, funcional y periodizado para corredores vs no funcional y no periodizado sobre el IMC, MG, MLG y DMO. Participaron un total de 18 corredores recreacionales sanos $(27,1$ años; IMC $=23,2 \mathrm{~kg} / \mathrm{m} 2$ ). Los sujetos se distribuyeron al azar en tres grupos: (i) entrenamiento WB-EMS funcional y periodizado para corredores, (ii) entrenamiento WB-EMS no funcional y no periodizado, y (iii) grupo control. La composición corporal se analizó por antropometría y bioimpedanciometría eléctrica determinándose el IMC, MG, MLG y DMO. Los resultados del estudio ponen de manifiesto que el entrenamiento con WB-EMS (independientemente de la modalidad) produjo descensos significativos en IMC y MG $(P<0,05)$, experimentándose una tendencia en términos de mejora de MLG sólo en el grupo WB-EMS funcional $(P=0,053)$ al comparar con el grupo control, no observándose cambios en DMO. Por lo tanto, podemos concluir que un programa de entrenamiento WB-EMS produce mejoras significativas en la composición corporal en corredores recreacionales entrenados, específicamente en IMC y MG, y clínicamente relevantes en MLG únicamente en el grupo WB-EMS funcional.

Palabras clave: WB-EMS, corredores, masa grasa corporal, masa libre de grasa, IMC.

\section{Abstract}

Whole-Body-Electromyostimulation (WB-EMS) has become a new training tendency but its effect on body composition has not been thoroughly studied in young trained population. The principal aim of this study was to determine the effect of two types of WB-EMS training modalities (specific, functional and periodized for runners vs non-specific, non-functional and non-periodized on body composition variables, as body mass index (BMI) fat mass (FM), fat free mass (FFM) and bone mineral density (BMD) in recreational trained runners during endurance training cessation and to compare the effects of two WB-EMS training modalities, functional vs non-functional on BMI, FM, FFM and BMI. 18 healthy recreational runners ( 27.1 years; $B M I=23.2 \mathrm{Kg} / \mathrm{m} 2$ ) were randomized in three groups: (i) functional WB-EMS, (ii) non-functional WB-EMS and (iii) control group. Body composition parameters were assessed by anthropometry and bio-impedance and the variables measured were BMI, FM, FFM and BMD. Both WB-EMS modalities produced a significant decreases in BMI and FM $(p<0.05)$ and it was shown a tendency in terms of FFM improvements only in functional WB-EMS group when compared with control group $(P=0.053)$. No changes were observed in BMD in any cases. Therefore, 6-week-WB-EMS training program produced significant improvements on body composition in recreational trained runners, specifically on BMI and FM. Clinically but not significantly improvements were also observed on FFM only in functional WB-EMS group.

Key words: WB-EMS, runners, fat body mass, fat free mass. 


\section{Introducción}

$\mathrm{E}^{\mathrm{s}}$ 1 desentrenamiento deportivo es un periodo temporal durante el cual se produce una pérdida parcial de las adaptaciones inducidas por el entrenamiento en respuesta a situaciones de entrenamiento insuficiente (Maldonado-Martín, Cámara, James, Fernández-López, y ArtetxeGezuraga, 2017). Estos periodos son relativamente frecuentes en la mayoría de las disciplinas deportivas (lesiones, periodos de transición, micro-ciclos de descarga, etc.).

Diversos estudios ponen de manifiesto la relación existente entre la composición corporal de un sujeto y su nivel de condición física, asociándose mayor nivel de condición física a menor porcentaje de masa grasa (MG) y, por tanto, a un mayor rendimiento en pruebas de resistencia (Bale, Bradbury, y Colley, 1986; Belli, Meireles, Costa, Ackermann, y Ca, 2016; Mattila, Tallroth, y Marttinen, 2007).

Periodos de desentrenamiento de 5 semanas producen incrementos de más de un $15 \%$ de MG en deportistas entrenados y más de un $2 \%$ de Índice de Masa Corporal (IMC) (MaldonadoMartín y col., 2017), induciéndose por tanto una posible pérdida del rendimiento. Por lo tanto, se hace necesario plantear estrategias preventivas en términos de mantenimiento y/o mejora de los niveles de composición corporal durante periodos en los que el volumen de entrenamiento tenga que verse reducido.

La electroestimulación integral de cuerpo completo (WB-EMS, Whole Body Electromyostimulation) es un sistema de entrenamiento capaz de estimular hasta 14-18 grupos musculares de forma simultánea abarcando una superficie de 2800 centímetros cuadrados (Filipovic y col., 2015). El entrenamiento con WB-EMS parece producir incrementos en la masa libre de grasa (MLG), condición física y adherencia a la práctica de actividad física, así como decrementos en MG en población sedentaria, (Kemmler y col., 2016; Kemmler, Bebenek, Engelke, y von Stengel, 2014; Kemmler y von Stengel, 2013) pero no se ha estudiado su influencia en adultos sanos que practican carrera de forma recreacional.

Por tanto, los objetivos del presente trabajo han sido: (i) determinar el efecto de dos programas de entrenamiento con WB-EMS sobre la composición corporal, medida a través del IMC, MG, MLG y densidad mineral ósea (DMO) en corredores recreacionales entrenados durante un periodo de cese parcial en su entrenamiento convencional y (ii) comparar el efecto de sendos programas de entrenamiento con WB-EMS entre sí, uno periodizado y funcional para corredores (WB-EMS-F) y otro no funcional y no periodizado, similar al aplicado en la mayoría de estudios que emplean WB-EMS (WB-EMS-NF) sobre la composición corporal en los mencionados sujetos.

\section{Método}

\section{Diseño experimental}

Estudio longitudinal con dos grupos experimentales: (i) WB-EMS-F ( $n=6$ ), (ii) WB-EMS-NF $(n=6)$ y (iii) un grupo control $(n=6)$.

Los participantes fueron asignados aleatoriamente en los tres grupos experimentales. Aquellos que se incluyeron en los grupos WB-EMS experimentaron un cese de su programa de entrenamiento convencional y se les instruyó a realizar seis sesiones de entrenamiento (1 por semana) con WB-EMS (Miha Bodytec, Augsburg, Alemania) y de forma complementaria una sesión/semana en la que se llevaron a cabo 20 minutos de carrera continua (10 minutos al 60\% tasa cardiaca de reserva y 10 minutos al $70 \%$ tasa cardiaca de reserva). El grupo control mantuvo la metodología de los entrenamientos que venían realizando en meses anteriores: 
Amaro-Gahete, F. J.; De la O, A.; Roriguez-Gonzalez, L.; Castillo, M. J., y Gutierrez, A. (2018). Impacto de dos modalidades de entrenamiento con electroestimulación global de cuerpo completo sobre la composición corporal en corredores recreacionales durante periodos de desentrenamiento deportivo. RICYDE. Revista internacional de ciencias del deporte, 53(14), 205-218. https://doi.org/10.5232/ricyde2018.05302

aeróbico extensivo con una intensidad de $60-70 \%$ de la tasa cardiaca de reserva, duración de 45-60 minutos y frecuencia de 2-3 días por semana controlada con un pulsómetro (Polar RS300X, POLAR, Kempele, Finland). La tasa de asistencia mínima a las sesiones de WB-EMS para completar el programa fue establecida en $>80 \%$.

Participantes

22 corredores recreacionales de entre 18 y 35 años se ofrecieron voluntarios para realizar el estudio. 4 participantes no lo completaron por incompatibilidad horaria y motivos laborales. El estudio cumplió con la Declaración de Helsinki y fue aprobado por el comité de ética de la Universidad de Granada. Los criterios de inclusión fueron; (A) hombres, de 18-35 años; (B) sujetos entrenados (mínimo 6 meses); (C) declarados aptos tras examen médico; (D) no variación del peso corporal mayor de $3 \mathrm{~kg}$ durante los últimos 6 meses.

\section{Procedimiento}

Las evaluaciones pre-post entrenamiento se realizaron en un único día e incluyeron la medida de peso, talla, y bioimpedanciometría eléctrica (ver Figura 1).

La medición del peso y talla se realizó mediante una balanza-tallímetro SECA 700 (SECA, Berlín, Alemania) con precisión de $0,1 \mathrm{~kg}$. Para evaluar la composición corporal se utilizó bioimpedancia eléctrica (Inbody 720, Biospace, Gateshead, Reino Unido), siguiendo las recomendaciones de Alvero-Cruz et al. (Alvero-Cruz, Ronconi, Fernández-Vázquez, \& Manzanido, 2011), obteniéndose como resultado una estimación de MG $(\mathrm{kg}), \% \mathrm{MG}, \mathrm{MLG}(\mathrm{kg})$ y DMO $\left(\mathrm{g} / \mathrm{cm}^{2}\right)$.

Adicionalmente se controló la cantidad de actividad física no programada mediante el cuestionario IPAQ (Lee, Macfarlane, Lam, y Stewart, 2011), así como la ingesta nutricional pre-post entrenamiento mediante recordatorios 24 horas ( 2 realizados en días laborables y 1 en festivo) (Gills, Baker, y Auld, 2017). 
Amaro-Gahete, F. J.; De la O, A.; Roriguez-Gonzalez, L.; Castillo, M. J., y Gutierrez, A. (2018). Impacto de dos modalidades de entrenamiento con electroestimulación global de cuerpo completo sobre la composición corporal en corredores recreacionales durante periodos de desentrenamiento deportivo. RICYDE. Revista internacional de ciencias del deporte, 53(14), 205-218. https://doi.org/10.5232/ricyde2018.05302

PRE-TEST

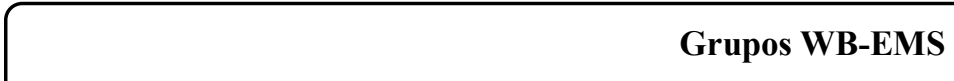

POST-TEST

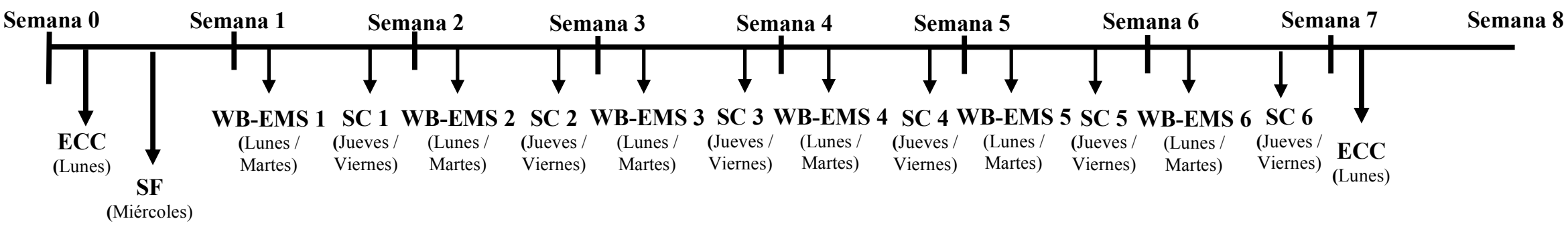

Figura 1. Línea temporal de acontecimientos relativos al programa de intervención.

ECC, Evaluación de la Composición Corporal; SF; Sesión de Familiarización; WB-EMS, Entrenamiento con Electroestimulación Global de Cuerpo Completo; SC, Sesión de Carrera. 
Amaro-Gahete, F. J.; De la O, A.; Roriguez-Gonzalez, L.; Castillo, M. J., y Gutierrez, A. (2018). Impacto de dos modalidades de entrenamiento con electroestimulación global de cuerpo completo sobre la composición corporal en corredores recreacionales durante periodos de desentrenamiento deportivo. RICYDE. Revista internacional de ciencias del deporte, 53(14), 205-218. https://doi.org/10.5232/ricyde2018.05302

\section{Protocolo de entrenamiento}

Los sujetos incluidos en los dos grupos WB-EMS realizaron una sesión a la semana con la metodología correspondiente a su respectivo grupo de intervención.

Los entrenamientos en ambos casos se caracterizaron por su corta duración, bajo volumen y alta intensidad; así mismo tuvo una progresión lineal en base a los parámetros eléctricos, el volumen, la carga y la intensidad para evitar posibles problemas de salud tales como un incremento excesivo de creatininfosfoquinasa (CPK) y rabdomiolisis (Finsterer y Stöllberger, 2015; Kemmler, Teschler, Bebenek, y von Stengel, 2015). Se controló la intensidad subjetiva del impulso eléctrico, mediante la escala de Borg (Borg, 1982). Previo al comienzo del programa de entrenamiento se realizó una sesión de familiarización en la que se aplicó WB-EMS por primera vez y se practicaron patrones motores básicos.

\section{Protocolo WB-EMS-F}

El mencionado protocolo se confeccionó en base a ejercicios específicos utilizados habitualmente para la mejora del rendimiento en corredores, aplicando los parámetros eléctricos adecuados a cada tipo de actividad (Filipovic, Kleinöder, Dörmann, y Mester, 2011). Los parámetros eléctricos utilizados siguieron las recomendaciones propuestas en otros estudios para mejorar la fuerza y la capacidad aeróbica (Amaro-Gahete, de la O, JuradoFasoli, Ruiz, y Gutiérrez, 2017; Filipovic, Kleinöder, Dörmann, y Mester, 2012). Se detallan en la tabla 1.

Las sesiones de entrenamiento se dividieron en cuatro partes (en todos los casos, los participantes realizaban el ejercicio propuesto cuando recibían impulso eléctrico): (A) Fase de calentamiento, (B) Fase de fuerza, (C) Fase de entrenamiento interválico de alta intensidad de Fuerza y (D) Fase de entrenamiento interválico de alta intensidad. La intensidad del impulso fue ajustada individualmente por percepción subjetiva cada 2 minutos. No se utilizó en ningún momento carga externa y se aplicó una metodología de entrenamiento en circuito. En la fase A, los participantes realizaron de 7-10 repeticiones ( 1 serie) de 3 ejercicios ([i] 1/2 sentadilla + curl bíceps, [ii] $1 / 2$ sentadilla y vuelos laterales, [iii] $1 / 2$ sentadilla y empuje horizontal); tanto la fase concéntrica como la excéntrica tuvieron una duración de 2 segundos. En la fase B, los participantes llevaron a cabo 1-2 series (5-10 repeticiones) de 6 ejercicios diferentes ([i] zancada estática + flexión de rodilla-cadera, [ii] swing americano, [iii] flexiones de brazos, [iv] sentadilla búlgara + empuje vertical, [v] 1/2 sentadilla sumo + elevaciones laterales, [vi] peso muerto + tracción horizontal); la fase concéntrica tuvo una duración de 1 segundo y la excéntrica de 3 segundos. En la fase $C$, los participantes realizaron 1 serie de 8 ejercicios ([i] frecuencia/cadencia de piernas en bipedestación con pierna derecha, [ii] frecuencia/cadencia de piernas en bipedestación con pierna izquierda, [iii] salto alternativo en step, [iv] frecuencia/cadencia de brazos sentado, [v] 1/2 sentadilla + salto, [vi] Escalador, [vii]) salto lateral en step, [viii] skipping), ejecutando el máximo número de repeticiones con una técnica correcta en 10 segundos (con 10 segundos de descanso entre ejercicios).

En la fase D, los participantes realizaron 1-2 series interválicas corriendo en tapiz rodante a dos intensidades diferentes; 65\% y 85\% del consumo máximo de oxígeno durante 30 segundos cada una. 
Amaro-Gahete, F. J.; De la O, A.; Roriguez-Gonzalez, L.; Castillo, M. J., y Gutierrez, A. (2018). Impacto de dos modalidades de entrenamiento con electroestimulación global de cuerpo completo sobre la composición corporal en corredores recreacionales durante periodos de desentrenamiento deportivo. RICYDE. Revista internacional de ciencias del deporte, 53(14), 205-218. https://doi.org/10.5232/ricyde2018.05302

\section{Protocolo WB-EMS-NF}

El protocolo de entrenamiento trató de replicar la metodología empleada en la mayoría de estudios experimentales previos realizados con WB-EMS (Kemmler y col., 2016; Kemmler y col., 2014; Kemmler y von Stengel, 2013); en la tabla 1 se adjunta la selección de los parámetros eléctricos utilizados.

Las sesiones se estructuraron en forma de circuito y se utilizaron 10 ejercicios dinámicos ([i] $1 / 2$ sentadilla + curl bíceps, [ii] peso muerto + tracción horizontal, [iii] $1 / 2$ sentadilla + flexión de tronco, [iv] 1/2 sentadilla + empuje vertical, [v] 1/2 sentadilla + elevaciones laterales, (vi) 1/2 sentadilla + empuje horizontal, [vii] $1 / 2$ sentadilla + elevaciones frontales, [viii] peso muerto + patada de tríceps ), [ix] 1/2 sentadilla + vuelos laterales, [x] rotación de tronco), semejantes a los establecidos en estudios anteriores. Se realizaron 6-10 series de cada ejercicio y la duración de la fase concéntrica y excéntrica fue de 2 segundos cada una. Se estableció un descanso entre series de 30 segundos. La intensidad del impulso fue ajustada individualmente por percepción subjetiva cada 2 minutos. 
Amaro-Gahete, F. J.; De la O, A.; Roriguez-Gonzalez, L.; Castillo, M. J., y Gutierrez, A. (2018). Impacto de dos modalidades de entrenamiento con electroestimulación global de cuerpo completo sobre la composición corporal en corredores recreacionales durante periodos de desentrenamiento deportivo. RICYDE. Revista internacional de ciencias del deporte, 53(14), 205-218. https://doi.org/10.5232/ricyde2018.05302

Tabla 1: Descripción de los parámetros eléctricos en las sesiones del grupo WB-EMS-F y WB-EMS-NF.

\begin{tabular}{|c|c|c|c|c|c|c|c|c|c|c|c|c|c|c|c|c|c|c|c|c|c|c|c|c|}
\hline \multirow[t]{2}{*}{ WB-EMS-F } & \multicolumn{4}{|c|}{ Sesión 1} & \multicolumn{4}{|c|}{ Sesión 2} & \multicolumn{4}{|c|}{ Sesión 3} & \multicolumn{4}{|c|}{ Sesión 4} & \multicolumn{4}{|c|}{ Sesión 5} & \multicolumn{4}{|c|}{ Sesión 6} \\
\hline & $\mathbf{C}$ & $\mathbf{F}$ & $\mathbf{H F}$ & HT & $\mathbf{C}$ & $\mathbf{F}$ & $\mathbf{H F}$ & HT & $\mathbf{C}$ & $\mathbf{F}$ & $\mathbf{H F}$ & HT & $\mathbf{C}$ & $\mathbf{F}$ & HF & HT & $\mathbf{C}$ & $\mathbf{F}$ & $\mathbf{H F}$ & HT & $\mathbf{C}$ & $\mathbf{F}$ & HF & HT \\
\hline $\begin{array}{c}\text { Duración total } \\
\text { (min) }\end{array}$ & 2 & 6 & 2 & 2 & 4 & 6 & 3 & 3 & 4 & 8 & 3 & 3 & 4 & 8 & 4 & 4 & 4 & 8 & 4 & 4 & 4 & 8 & 4 & 4 \\
\hline $\begin{array}{c}\text { Frecuencia } \\
(\mathrm{Hz})\end{array}$ & 12 & 55 & 60 & 20 & 12 & 65 & 70 & 25 & 12 & 75 & 80 & 35 & 12 & 85 & 90 & 40 & 12 & 85 & 90 & 40 & 12 & 85 & 90 & 40 \\
\hline $\begin{array}{c}\text { Ancho de impulso } \\
(\mu \mathrm{s})\end{array}$ & 150 & 175 & 200 & 150 & 175 & 225 & 250 & 200 & 175 & 275 & 300 & 250 & 200 & 350 & 350 & 300 & 200 & 350 & 350 & 300 & 200 & 350 & 350 & 300 \\
\hline $\begin{array}{c}\text { Intensidad de impulso } \\
(\mathrm{mA})\end{array}$ & 100 & 50 & 50 & 80 & 100 & 55 & 55 & 80 & 100 & 55 & 60 & 90 & 100 & 60 & 60 & 90 & 100 & 60 & 60 & 90 & 100 & 60 & 60 & 90 \\
\hline $\begin{array}{l}R P E \\
(6-20)\end{array}$ & 10 & 12 & 13 & 13 & 10 & 12 & 13 & 13 & 10 & 14 & 15 & 15 & 10 & 16 & 17 & 17 & 10 & 16 & 17 & 17 & 10 & 16 & 17 & 17 \\
\hline Ciclo de trabajo & 50 & 50 & 50 & 50 & 50 & 50 & 50 & 50 & 50 & 50 & 50 & 50 & 50 & 50 & 50 & 50 & 50 & 50 & 50 & 50 & 50 & 50 & 50 & 50 \\
\hline$(\%)$ & $4: 4$ & $4: 4$ & $10: 10$ & $30: 30$ & $4: 4$ & $4: 4$ & $10: 10$ & $30: 30$ & $4: 4$ & $4: 4$ & $10: 10$ & $30: 30$ & $4: 4$ & $4: 4$ & $10: 10$ & $30: 30$ & $4: 4$ & $4: 4$ & 10:10 & $30: 30$ & $4: 4$ & $4: 4$ & $10: 10$ & $30: 30$ \\
\hline $\begin{array}{c}\text { Duración total } \\
\text { (min) }\end{array}$ & \multicolumn{4}{|c|}{12} & \multicolumn{4}{|c|}{16} & \multicolumn{4}{|c|}{18} & \multicolumn{4}{|c|}{20} & \multicolumn{4}{|c|}{20} & \multicolumn{4}{|c|}{20} \\
\hline $\begin{array}{c}\text { Frecuencia } \\
(\mathrm{Hz})\end{array}$ & \multicolumn{4}{|c|}{85} & \multicolumn{4}{|c|}{85} & \multicolumn{4}{|c|}{85} & \multicolumn{4}{|c|}{85} & \multicolumn{4}{|c|}{85} & \multicolumn{4}{|c|}{85} \\
\hline $\begin{array}{c}\text { Ancho de impulso } \\
(\mu \mathrm{s})\end{array}$ & \multicolumn{4}{|c|}{350} & \multicolumn{4}{|c|}{350} & \multicolumn{4}{|c|}{350} & \multicolumn{4}{|c|}{350} & & & 50 & & & & 50 & \\
\hline $\begin{array}{c}\text { Intensidad de impulso } \\
(\mathrm{m} A)\end{array}$ & & & & & & & & & & & 0 & & & & 60 & & & & 0 & & & & 50 & \\
\hline $\begin{array}{c}R P E \\
(6-20)\end{array}$ & & & & & & & & & & & -16 & & & & -17 & & & & -17 & & & & -17 & \\
\hline $\begin{array}{c}\text { Ciclo de trabajo } \\
(\%)\end{array}$ & & $50 \%$ & $(4: 4)$ & & & $50 \%$ & $(4: 4)$ & & & $50 \%$ & $(4: 4)$ & & & $50 \%$ & $(4: 4)$ & & & $50 \%$ & $(4: 4)$ & & & $50^{\circ}$ & $(4: 4)$ & \\
\hline
\end{tabular}

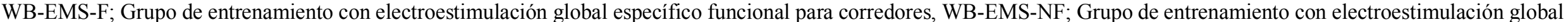

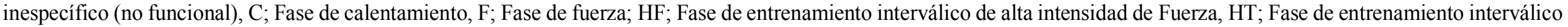
de alta intensidad, RPE; Percepción subjetiva del esfuerzo, Hz; Hercios, $\mu$ s; Microsegundos, mA; Miliamperios 
Amaro-Gahete, F. J.; De la O, A.; Roriguez-Gonzalez, L.; Castillo, M. J., y Gutierrez, A. (2018). Impacto de dos modalidades de entrenamiento con electroestimulación global de cuerpo completo sobre la composición corporal en corredores recreacionales durante periodos de desentrenamiento deportivo. RICYDE. Revista internacional de ciencias del deporte, 53(14), 205-218. https://doi.org/10.5232/ricyde2018.05302

\section{Análisis estadístico}

Los resultados se expresan mediante media y desviación típica $(\mathrm{X} \pm \mathrm{DS})$. Los supuestos de normalidad y homocedasticidad se comprobaron respectivamente con el test de ShapiroWilks y el test de Levene.

Para valorar los cambios pre-post entrenamiento en las variables dependientes se aplicó el test T-Student para muestras apareadas considerando un intervalo de confianza al 95\%.

Para examinar el efecto del programa de entrenamiento sobre la composición corporal (IMC, MG, MLG y DMO) se utilizó un análisis de la covarianza de una vía ajustando por los valores iniciales de la respectiva variable estudiada. Las comparaciones entre grupos fueron ajustadas por Bonferroni. El análisis estadístico se realizó en su totalidad a través del paquete estadístico SPSS (versión 20, IBM, Ehningen, Alemania), estableciendo el nivel de significación $\mathrm{p}<0,05$.

\section{Resultados}

Los participantes del grupo WB-EMS-F tuvieron una tasa de asistencia a las sesiones de entrenamiento del 95,83\%, y la del grupo WB-EMS-NF fue del 97,31\%; no se registraron efectos adversos con ninguno de los dos programas y no se encontraron diferencias en términos de actividad física realizada fuera del programa de entrenamiento ni en ingesta calórica y/o distribución de macronutrientes (pre-post entrenamiento). El grupo control realizó un total de 2,68 sesiones de media por semana promediando un total de 52 minutos por sesión a una intensidad media del $63 \%$ de la tasa cardiaca de reserva.

Las características descriptivas, los valores pre-test y post-test relativos a la composición corporal y la diferencia de medias en los grupos WB-EMS-F, WB-EMS-NF y del grupo control se presentan en la tabla 2.

El análisis de la covarianza reveló que los sujetos del grupo WB-EMS-F y los del grupo WBEMS-NF disminuyeron su IMC (-0,54 $\pm 0,40$ vs $\left.-0,32 \pm 0,24 \mathrm{~kg} / \mathrm{m}^{2}\right)$, mientras que en el grupo control se produjo un ligero incremento no significativo $\left(0,14 \pm 0,15 \mathrm{~kg} / \mathrm{m}^{2}\right)$. Tras ajustar por los valores pre-test (Figura 2A), se observaron diferencias estadísticamente significativas entre el grupo WB-EMS-F y el grupo control $\left(-0,68[-1.12,-0,23] \mathrm{kg} / \mathrm{m}^{2} ; \mathrm{P}<0,01\right)$, y también entre el grupo WB-EMS-NF y el grupo control $\left(-0,46[-0,91,-0,02] \mathrm{kg} / \mathrm{m}^{2} ; \mathrm{P}<0,05\right)$. No se observaron diferencias estadísticamente significativas en relación al IMC entre los grupos WB-EMS-F y WB-EMS-NF. 
Amaro-Gahete, F. J.; De la O, A.; Roriguez-Gonzalez, L.; Castillo, M. J., y Gutierrez, A. (2018). Impacto de dos modalidades de entrenamiento con electroestimulación global de cuerpo completo sobre la composición corporal en corredores recreacionales durante periodos de desentrenamiento deportivo. RICYDE. Revista internacional de ciencias del deporte, 53(14), 205-218. https://doi.org/10.5232/ricyde2018.05302

Tabla 2: Parámetros descriptivos y cambios en las variables principales y secundarias después de programa de entrenamiento de 6 semanas.

\begin{tabular}{|c|c|c|c|c|c|c|}
\hline & \multicolumn{2}{|c|}{ WB-EMS-F $(n=6)$} & \multicolumn{2}{|c|}{ WB-EMS-NF $(n=6)$} & \multicolumn{2}{|c|}{ GRUPO CONTROL $(n=6)$} \\
\hline & PRE & POST & PRE & POST & PRE & POST \\
\hline PESO (kg) & $70,1 \pm 11,1$ & $68,6 \pm 11,0$ & $73,8 \pm 9,8$ & $72,7 \pm 9,3$ & $73,6 \pm 2,1$ & $74,1 \pm 1,9$ \\
\hline $\mathrm{IMC}\left(\mathrm{kg} / \mathrm{m}^{2}\right)$ & $22,6 \pm 2,8$ & $22,2 \pm 2,6$ & $24,5 \pm 3,3$ & $24,1 \pm 3,2$ & $23,4 \pm 0,8$ & $23,5 \pm 0,7$ \\
\hline MG (kg) & $12,3 \pm 1,3$ & $10,9 \pm 1,1$ & $13,2 \pm 2,1$ & $12,1 \pm 1,81$ & $12,1 \pm 2,2$ & $12,2 \pm 2,3$ \\
\hline MG (\%) & $17,6 \pm 2,7$ & $15,9 \pm 3,0$ & $17,8 \pm 3,6$ & $16,6 \pm 3,3$ & $16,3 \pm 4,1$ & $16,5 \pm 4,4$ \\
\hline MLG (kg) & $33,4 \pm 3,2$ & $34,7 \pm 3,2$ & $35,8 \pm 4,6$ & $36,2 \pm 4,1$ & $37,3 \pm 1,9$ & $37,2 \pm 2,0$ \\
\hline $\operatorname{DMO}\left(\mathrm{g} / \mathrm{cm}^{2}\right)$ & $3,2 \pm 0,3$ & $3,3 \pm 0,3$ & $3,5 \pm 0,5$ & $3,6 \pm 0,5$ & $3,6 \pm 0,4$ & $3,6 \pm 0,5$ \\
\hline
\end{tabular}

Los valores se expresan como media \pm desviación típica (valores pre-post entrenamiento), $\mathrm{y}$ diferencia de medias $-\bar{X}_{1}-\bar{X}_{2}$ e intervalo de confianza al $95 \%$ - IC (95\%), a menos que se indique lo contrario. Nivel de significación pre-post entrenamiento: $* \mathrm{P}<0,05 * * \mathrm{P}<0,01 * * *$ $\mathrm{P}<0,001$.

Abreviaturas: IMC; Índice de Masa Corporal, MG; Masa Grasa, MLG; Masa Libre de Grasa, DMO; Densidad Mineral Ósea. WB-EMS-F; Entrenamiento con electroestimulación de cuerpo completo funcional para corredores, WB-EMS-NF; Entrenamiento con electroestimulación de cuerpo completo no funcional (inespecífico). 
Amaro-Gahete, F. J.; De la O, A.; Roriguez-Gonzalez, L.; Castillo, M. J., y Gutierrez, A. (2018). Impacto de dos modalidades de entrenamiento con electroestimulación global de cuerpo completo sobre la composición corporal en corredores recreacionales durante periodos de desentrenamiento deportivo. RICYDE. Revista internacional de ciencias del deporte, 53(14), 205-218. https://doi.org/10.5232/ricyde2018.05302

A

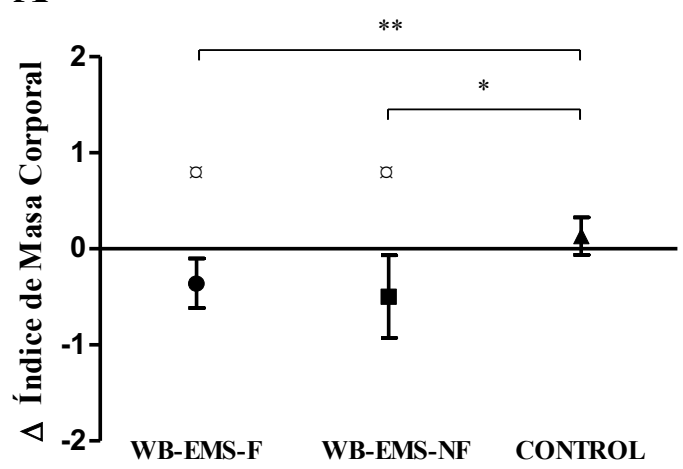

B

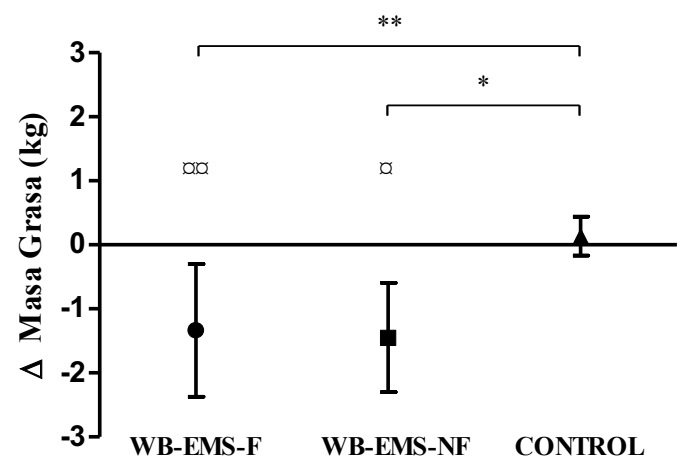

C

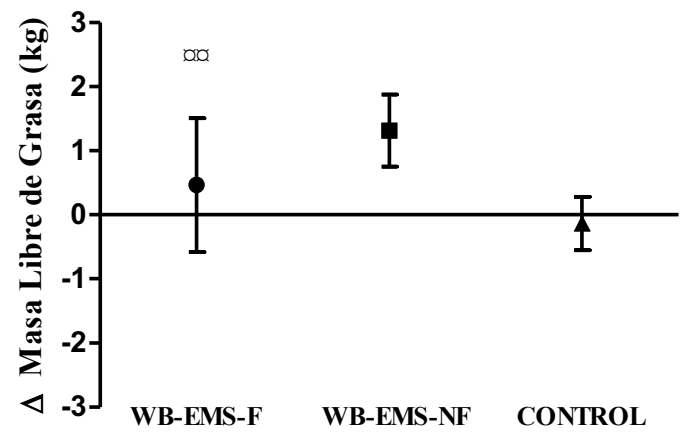

D

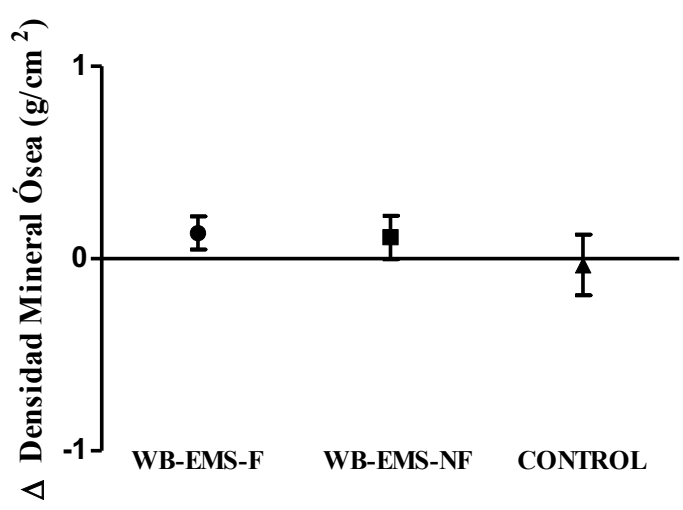

Figura 2: Diferencia de medias \pm DS (95\% IC) en las variables dependientes principales tras el programa de intervención. WB-EMS-F; Grupo Electroestimulación Integral funcional para corredores, WB-EMS-NF; Entrenamiento con electroestimulación de cuerpo completo Integral no funcional. Nivel de significación prepost entrenamiento: $\propto \mathrm{P}<0,05$ a $\mathrm{P}<0,01$ a $\infty \mathrm{P}<0,001$. Nivel de significación entre grupos: ${ }^{*} \mathrm{P}<0,05 * * \mathrm{P}<0,01$ $* * * \mathrm{P}<0,001$. 
Amaro-Gahete, F. J.; De la O, A.; Roriguez-Gonzalez, L.; Castillo, M. J., y Gutierrez, A. (2018). Impacto de dos modalidades de entrenamiento con electroestimulación global de cuerpo completo sobre la composición corporal en corredores recreacionales durante periodos de desentrenamiento deportivo. RICYDE. Revista internacional de ciencias del deporte, 53(14), 205-218. https://doi.org/10.5232/ricyde2018.05302

La masa grasa total (Figura 2B) se redujo en los grupos WB-EMS-F y WB-EMS-NF (X \pm DS, $-1,45 \pm 0,81$ y $-1,20 \pm 0,99 \mathrm{~kg}$ ); sin embargo, aumentó mínimamente en el grupo control ( $\mathrm{X} \pm \mathrm{DS}, 0,15 \pm 0,28 \mathrm{~kg}$ ). Al comparar los grupos entre sí, se observaron diferencias estadísticamente significativas entre los grupos WB-EMS-F y WB-EMS-NF respecto al grupo control ( $\triangle \mathrm{X},-1,54$ y $-1,29 \mathrm{~kg} ; 95 \% \mathrm{IC},-2,68,-0,41$ y $-2,46,0,12 \mathrm{~kg} ; \mathrm{P}<0,01$ у $\mathrm{P}<0,05)$. No hubo diferencias estadísticamente significativas entre los dos grupos experimentales, pese a que en el grupo WB-EMS-F existen indicios de reducción de MG ligeramente superiores teniendo en cuenta el tamaño de muestra $(\Delta \mathrm{X},-0,26 \mathrm{~kg} ; 95 \% \mathrm{IC},-1,41,0,89 \mathrm{~kg} ; \mathrm{P}=0,11)$.

En la tabla 2, se puede contemplar un aumento de masa libre de grasa de los grupos WBEMS-F y WB-EMS-NF (X $\pm \mathrm{DS}, 1,31 \pm 0,54 \mathrm{vs} 0,47 \pm 0,99 \mathrm{~kg}$ ); no hubo cambios significativos en el grupo control. Al realizar las comparaciones entre grupos (Figura 2C), ninguna de ellas fue estadísticamente significativa, existiendo únicamente indicios de significación en la comparación del grupo WB-EMS-F con el grupo control $(\Delta \mathrm{X}, 1,19 \mathrm{~kg} ; 95 \% \mathrm{IC},-0,07,2,38$ $\mathrm{kg} ; \mathrm{P}=0,053)$.

No se produjeron cambios estadísticamente significativos en DMO (pre y post) en ninguno de los grupos que forman parte del estudio (Figura 2D) WB-EMS-F, WB-EMS-NF y grupo control (X $\pm \mathrm{DS}, 0,13 \pm 0,08,0,11 \pm 0,11,0,03 \pm 0,15 \mathrm{~kg})$.

\section{Discusión}

Tras analizar los resultados relativos al estudio, se observó un descenso en el IMC y la MG y un aumento en la MLG en los sujetos que se sometieron a un programa de entrenamiento con WB-EMS independiente de la modalidad de entrenamiento (WB-EMS-F y WB-EMS$\mathrm{NF}$ ) en comparación con el grupo control. No se observaron cambios en la DMO, hecho que resulta evidente ya que este parámetro no es sensible al cambio en tan sólo 6 semanas.

Los resultados ponen de manifiesto que los grupos que realizaron entrenamiento con WBEMS redujeron significativamente su IMC, WB-EMS-F (-3,09\%) y WB-EMS-NF (-2,91\%); estos resultados coinciden con los obtenidos en otro estudio realizado recientemente en mujeres sedentarias $(-2,69 \%)$ tras llevar a cabo 14 semanas de entrenamiento (Kemmler, Schliffka, Mayhew, y von Stengel, 2010); el incremento podría deberse al aumento del gasto energético en reposo post-ejercicio, ya que tal y como ponen de manifiesto De la $\mathrm{O}$ y col., (De la O, Amaro, Roero, y Gutiérrez, 2015) éste se mantiene elevado hasta 72 horas después de la realización de una sesión con WB-EMS, generando un mayor gasto energético.

Respecto a la MG, se observó un descenso tanto en el grupo WB-EMS-F (-9,65\%), como en el WB-EMS-NF (-6,74\%), sin cambios significativos en el grupo control $(0,8 \%)$. Estos resultados confirman los obtenidos por Kemmler y col. (2016), los cuales observaron una reducción del porcentaje de MG tras la aplicación de un programa de intervención con WBEMS $(3,73 \%)$ y HIIT $(4,41 \%)$ de seis semanas, no encontrándose diferencias significativas entre ellos, pero sí respecto al grupo control (Kemmler y col., 2016). Otro estudio de 14 semanas de duración, observó descensos en el porcentaje de $\mathrm{MG}(-8,61 \%)$ respecto al pretest (Kemmler y col., 2010). En el caso de programas con una duración de carácter anual (5456 semanas), la reducción en relación a esta variable es también altamente significativa, $12,68 \%$ (Kemmler y von Stengel, 2013) y -8,01\% (Kemmler y col., 2014). 
Amaro-Gahete, F. J.; De la O, A.; Roriguez-Gonzalez, L.; Castillo, M. J., y Gutierrez, A. (2018). Impacto de dos modalidades de entrenamiento con electroestimulación global de cuerpo completo sobre la composición corporal en corredores recreacionales durante periodos de desentrenamiento deportivo. RICYDE. Revista internacional de ciencias del deporte, 53(14), 205-218. https://doi.org/10.5232/ricyde2018.05302

El grupo WB-EMS-F y el grupo WB-EMS-NF muestran incrementos en relación a la MLG $(3,89 \%$ y $1,12 \%)$ respecto al grupo control que mantiene constantes los valores del pre-test $(-0,09 \%)$. Kemmler et al. (2016) con un programa de intervención de la misma duración observó un incremento de $1,63 \%$ y de un 1,81\% con un programa HIIT en el mismo periodo temporal (Kemmler \& col., 2016). Así mismo, estudios de mayor duración (54 y 56 semanas) obtienen incrementos de 3,17\% (Kemmler \& von Stengel, 2013) y 3,54\% (Kemmler \& col., 2014).

No se observaron variaciones estadísticamente significativas de la DMO en los grupos experimentales WB-EMS-F (0,42\%) y WB-EMS-NF $(0,54 \%)$ y grupo control $(0,28 \%)$. Estos resultados son coherentes con los obtenidos por Von Stengel et tal. (2015), los cuales no observan cambios tras la aplicación de un programa de intervención con WB-EMS $(0,28 \%$ en columna dorsal y 0,12\% en cadera) (von Stengel, Bebenek, Engelke, y Kemmler, 2015).

El estudio cuenta con las siguientes limitaciones: (i) no se ha tenido en cuenta un grupo experimental que realice un programa de ejercicio similar en términos de volumen de entrenamiento y selección de ejercicios a los propuestos en el estudio actual pero con ausencia de WB-EMS, por lo que no se puede atribuir el efecto obtenido en términos de composición corporal únicamente y con total seguridad a esta metodología de entrenamiento, (ii) no se han controlado variables relativas a parámetros sanguíneos que controlen la existencia o no de altos niveles de CPK y rabdomiolisis, (iii) no se ha establecido un control de la actividad física realizada por los sujetos de forma objetiva (acelerometría) pero sí subjetiva mediante el cuestionario IPAQ (Lee y col., 2011), (iv) el número de sujetos por grupo es limitado y (v) los datos deberían ser corroborados en participantes de ambos sexos, con otros rangos de edad y en otras modalidades deportivas.

\section{Conclusión}

En conclusión, los resultados del estudio sugieren que un programa de entrenamiento WBEMS mejora la composición corporal produciendo un descenso del IMC y MG e incrementando MLG en corredores recreaciones respecto a un grupo control. Así mismo, un programa de entrenamiento WB-EMS funcional para corredores no difiere significativamente en términos de mejora la composición corporal respecto a uno no funcional, pero parece existir una tendencia en beneficio del grupo WB-EMS-F en términos de MG siendo dichas diferencias clínicamente relevantes. Se hacen necesarios más estudios que confirmen estos resultados y profundicen en aclarar el mecanismo causante del efecto que se muestra.

\section{Referencias}

Alvero-Cruz, J.; Ronconi, M.; Fernández-Vázquez, R., \& Manzanido, J. (2011). Bioelectrical impedance analysis as a method of body composition estimation: a practical approach. Revista Andaluza de Medicina del Deporte, 4(4), 167-74.

Bale, P.; Bradbury, D., \& Colley, E. (1986). Anthropometric and training variables related to $10 \mathrm{~km}$ running performance. British Journal of Sports Medicine, 20(4), 170-3. https://doi.org/10.1136/bjsm.20.4.170 
Amaro-Gahete, F. J.; De la O, A.; Roriguez-Gonzalez, L.; Castillo, M. J., y Gutierrez, A. (2018). Impacto de dos modalidades de entrenamiento con electroestimulación global de cuerpo completo sobre la composición corporal en corredores recreacionales durante periodos de desentrenamiento deportivo. RICYDE. Revista internacional de ciencias del deporte, 53(14), 205-218. https://doi.org/10.5232/ricyde2018.05302

Belli, T.; Meireles, C.; Costa, M.; Ackermann, M., \& Ca, G. (2016). Somatotype, body composition and performance in ultramarathon. Revista Brasileira de Cineantropometria y Desempenho Humano, 18(2), 127-153.

Borg, G. A. (1982). Psychophysical bases of perceived exertion. Medicine and science in sports and exercise, 14(5), 377-381.

https://doi.org/10.1249/00005768-198205000-00012

Filipovic, A.; Kleinöder, H.; Dörmann, U., \& Mester, J. (2011). Electromyostimulation-a systematic review of the influence of training regimens and stimulation parameters on effectiveness in electromyostimulation training of selected strength parameters. Journal of Strength and Conditioning Research, 25(11), 3218-38. https://doi.org/10.1519/JSC.0b013e318212e3ce

Filipovic, A.; Kleinöder, H.; Plück, D.; Hollmann, W.; Bloch, W., \& Grau, M. (2015). Influence of Whole-Body Electrostimulation on Human Red Blood Cell Deformability. Journal of Strength and Conditioning Research, 29(9), 2570-8. https://doi.org/10.1519/JSC.0000000000000916

Finsterer, J., \& Stöllberger, C. (2015). Severe rhabdomyolysis after MIHA-bodytec $®$ electrostimulation with previous mild hyper-CK-emia and noncompaction. International Journal of Cardiology, 180, 100-2. https://doi.org/10.1016/j.ijcard.2014.11.148

Gills, S.; Baker, S., \& Auld, G. (2017). Collection Methods for the 24-Hour Dietary Recall as Used in the Expanded Food and Nutrition Education Program. Journal of Nutrition Education and Behavior, 49(3), 250-256.e1. https://doi.org/10.1016/j.jneb.2016.10.009

Kemmler, W.; Bebenek, M.; Engelke, K., \& von Stengel, S. (2014). Impact of wholebody electromyostimulation on body composition in elderly women at risk for sarcopenia: The Training and ElectroStimulation Trial (TEST-III). Age, 36(1), 395406. https://doi.org/10.1007/s11357-013-9575-2

Kemmler, W.; Schliffka, R.; Mayhew, J. L., \& von Stengel, S. (2010). Effects of wholebody electromyostimulation on resting metabolic rate, body composition, and maximum strength in postmenopausal women: The Training and ElectroStimulation Trial. Journal of Strength and Conditioning Research, 24(7), 1880-7. https://doi.org/10.1519/JSC.0b013e3181ddaeee

Kemmler, W., \& von Stengel, S. (2013). Whole-body electromyostimulation as a means to impact muscle mass and abdominal body fat in lean, sedentary, older female adults: subanalysis of the TEST-III trial. Clinical Interventions in Aging, 8, 1353-64. https://doi.org/10.2147/CIA.S52337

Kemmler, W.; Teschler, M.; Bebenek, M., \& von Stengel, S. (2015). (Very) high Creatinkinase concentration after exertional whole-body electromyostimulation application: health risks and longitudinal adaptations. Wiener Medizinische Wochenschrift, 165(21-22), 427-35. https://doi.org/10.1007/s10354-015-0394-1

Kemmler, W.; Teschler, M.; Weißenfels, A.; Bebenek, M.; Fröhlich, M.; Kohl, M., \& von Stengel, S. (2016). Effects of Whole-Body Electromyostimulation versus HighIntensity Resistance Exercise on Body Composition and Strength: A Randomized Controlled Study. Complementary and Alternative Medicine, 1, 1-9. https://doi.org/10.1155/2016/9236809 
Amaro-Gahete, F. J.; De la O, A.; Roriguez-Gonzalez, L.; Castillo, M. J., y Gutierrez, A. (2018). Impacto de dos modalidades de entrenamiento con electroestimulación global de cuerpo completo sobre la composición corporal en corredores recreacionales durante periodos de desentrenamiento deportivo. RICYDE. Revista internacional de ciencias del deporte, 53(14), 205-218. https://doi.org/10.5232/ricyde2018.05302

De la O, A.; Amaro, F.; Roero, C.; y Gutiérrez, A. (2015). Influencia de tres tipos diferentes de entrenamiento (Electroestimulación global, High Intensity Interval Training (HIIT) y Aerobio convencional) sobre el metabolismo basal post esfuerzo. Revista Andaluza de Medicina del Deporte, 8(1), 27. https://doi.org/10.1016/j.ramd.2014.10.020

Lee, P.; Macfarlane, D.; Lam, T., \& Stewart, S. (2011). Validity of the international physical activity questionnaire short form (IPAQ-SF): A systematic review. International Journal of Behavioral Nutrition and Physical Activity, 8(1), 115-121. https://doi.org/10.1186/1479-5868-8-115

Maldonado-Martín, S.; Cámara, J.; James, D.; Fernández-López, J., \& Artetxe-Gezuraga, $X$. (2017). Effects of long-term training cessation in young top-level road cyclists. Journal of Sports Sciences, 35(14), 1396-1401. https://doi.org/10.1080/02640414.2016.1215502

Mattila, V.; Tallroth, K., \& Marttinen, M. (2007). Physical fitness and performance. Body composition by DEXA and its association with physical fitness in 140 conscripts. Medicine and Science, 39(12), 2242-7.

von Stengel, S.; Bebenek, M.; Engelke, K., \& Kemmler, W. (2015). Whole-Body Electromyostimulation to Fight Osteopenia in Elderly Females: The Randomized Controlled Training and Electrostimulation Trial (TEST-III). Journal of Osteoporosis, 20, 520-543.

https://doi.org/10.1155/2015/643520 